\title{
Energetic particle parallel diffusion in a cascading wave turbulence in the foreshock region
}

\author{
F. Otsuka ${ }^{1}$, Y. Omura ${ }^{1}$, and O. Verkhoglyadova ${ }^{1,2}$ \\ ${ }^{1}$ Research Institute for Sustainable Humanosphere, Kyoto University, Gokasho, Uji, Kyoto, 611-0011, Japan \\ ${ }^{2}$ Institute of Geophysics and Planetary Physics, University of California, Riverside, CA 92521, USA
}

Received: 11 May 2007 - Revised: 27 August 2007 - Accepted: 27 August 2007 - Published: 7 September 2007

\begin{abstract}
We study parallel (field-aligned) diffusion of energetic particles in the upstream of the bow shock with test particle simulations. We assume parallel shock geometry of the bow shock, and that MHD wave turbulence convected by the solar wind toward the shock is purely transverse in one-dimensional system with a constant background magnetic field. We use three turbulence models: a homogeneous turbulence, a regular cascade from a large scale to smaller scales, and an inverse cascade from a small scale to larger scales. For the homogeneous model the particle motions along the average field are Brownian motions due to random and isotropic scattering across 90 degree pitch angle. On the other hand, for the two cascade models particle motion is non-Brownian due to coherent and anisotropic pitch angle scattering for finite time scale. The mean free path $\lambda_{\|}$ calculated by the ensemble average of these particle motions exhibits dependence on the distance from the shock. It also depends on the parameters such as the thermal velocity of the particles, solar wind flow velocity, and a wave turbulence model. For the inverse cascade model, the dependence of $\lambda_{\|}$at the shock on the thermal energy is consistent with the hybrid simulation done by Giacalone (2004), but the spatial dependence of $\lambda_{\|}$is inconsistent with it.
\end{abstract}

\section{Introduction}

Spatial diffusion process plays a central role in propagation and diffusive shock acceleration (DSA) of energetic particles. Charged particles are scattered by magnetohydorodynamic (MHD) waves in parallel and perpendicular directions to the ambient magnetic field line. The former is referred to here as parallel (field-aligned) diffusion, and the latter as perpendicular (cross-field) diffusion. In this paper we study

Correspondence to: F. Otsuka

(f-otsuka@ rish.kyoto-u.ac.jp) the parallel diffusion of energetic particles in the MHD turbulence in order to apply our study to the DSA process in the parallel shock geometry. Fundamentally, parallel diffusion is described as a consequence of pitch angle diffusion. Namely, frequent and random scattering through 90 degree pitch angle occurs as a consequence of pitch angle diffusion by MHD waves. Accordingly, particles travel back and forth along the mean field, resulting in the random walk and the parallel diffusion.

In quasi-linear theory (QLT), pitch angle diffusion coefficient is expressed as a function of power of magnetic field fluctuation for the resonant wave (Kennel and Petschek, 1966; Tsurutani and Lakhina, 1997; Jokipii, 1966; Lee, 1982; Gordon et al., 1999). The pitch angle relaxation time $\tau$, at which the pitch angle distribution reaches a near-isotropic equilibrium, is defined by the reciprocal pitch angle diffusion coefficient. Then, for a much longer time scale than $\tau$, the mean free path is approximated as $\lambda_{\|} \sim v_{t h} \tau$, where $v_{t h}$ is thermal velocity of particles (e.g., Schlickeiser, 2002; Tsurutani et al., 2002). However, it is a long standing problem that the observed mean free paths have some discrepancies from the prediction of the QLT in a one-dimensional slab turbulence model. The QLT underestimates absolute values of the observed $\lambda_{\|}$by more than an order of magnitude (Tsurutani et al., 2002; Droge, 2000). Researchers have discussed this problem by using a so-called composite slab/two-dimensional geometry for the MHD turbulence model (Bieber et al., 1994). In this paper, we focus our discussion on the effect of spatially evolving MHD waves using a one-dimensional model. Consequently, we find that the obtained $\lambda_{\|}$has a spatial dependence, and that $\lambda_{\|}$at the shock is found to be larger than the value in the QLT.

It is evident from multi-spacecraft observations of energetic particles that the mean free path $\lambda_{\|}$has a spatial dependence due to variations of the magnetic field turbulence level, power spectrum of the waves, and correlation length of the waves (Beeck et al., 1987). However, the relationship

Published by Copernicus Publications on behalf of the European Geosciences Union and the American Geophysical Union. 


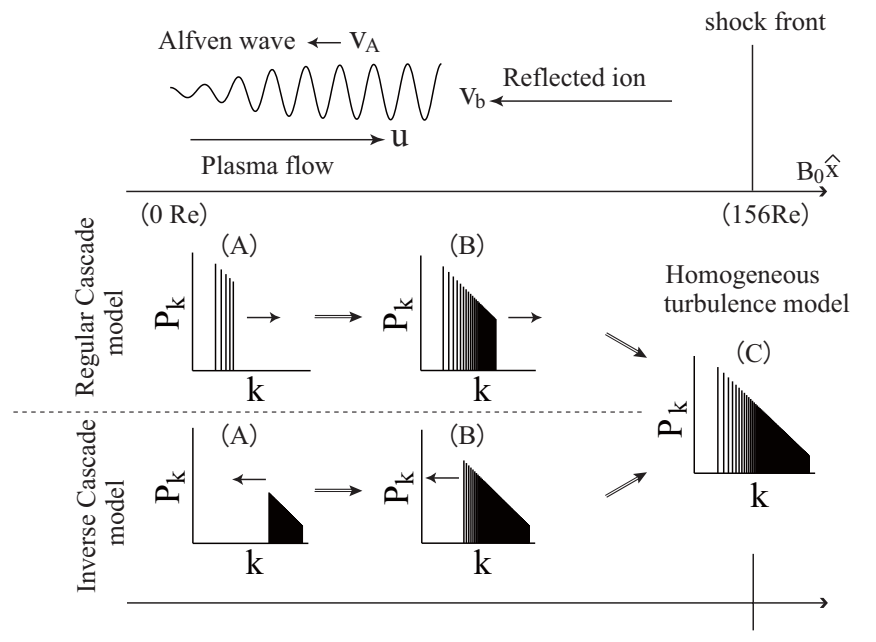

Fig. 1. Schematic Picture of the present model. Upper panel shows wave generation mechanism via ion beam instability. Lower panels show the spatial evolutions of the power spectrum for the MHD waves in two cascade models. Lebels (A), (B), and (C) refer to spectra at different distances from the bow shock (see Fig. 2 for details)

between the spatial dependence of $\lambda_{\|}$and statistics of the magnetic field turbulence is not clear. In numerical models for particle accelerations at a quasi-parallel interplanetary shock (Zank et al., 2000; Li et al., 2003, 2005), the spatial dependence is empirically introduced by index $\beta$ as a free parameter, $\lambda_{\|} \sim p^{\alpha} r^{\beta}$, where $r$ is radial distance from the sun, $p$ is particle momentum, and $\alpha$ is an index of the momentum dependence. We find that the model based on the QLT is not sufficient for description of the relationship between spatial variations of the mean free path and the statistics of MHD waves.

Nonlinear evolution of MHD waves has been observed in the so-called shocklets, short large-amplitude magnetic structures (so-called SLAMS) in the interplanetary space and in the Earth's foreshock region (Hoppe et al., 1981; Tsurutani et al., 1990, 2005a). The magnetic field turbulence near the Earth's bow shock has intermittent properties caused by nonlinear interactions among waves (Koga et al., 2007). Also, magnetic decreases (MDs) and magnetic holes (MHs) have important consequences for the generation of high frequency turbulence in the interplanetary space (Tsurutani et al., 2005a,b). In this paper, we model one-dimensional wave cascading upstream of a parallel shock for spatially evolving MHD waves. Fluctuations of the transverse fields are given by superposition of sinusoidal waves with different wavenumbers and random phases, and the ambient magnetic field magnitude is assumed constant. Thus, the magnetic field models used in this paper cannot describe wave steepening, MDs, MHs, and intermittency in the field turbulence, which, however, may have significant contributions to the particle scattering (see Sect. 3.2 in Tsurutani et al. (2005a)).
We employ two cascade models of MHD waves convected by the solar wind toward the shock: cascading to small scales (regular cascade model) and cascading to large scales (inverse cascade model). Additionally, a statistically homogeneous turbulence model is employed for comparison with the two cascade models. The schematic pictures of the two cascade models are shown in Fig. 1. We assume that Alfvén waves, excited far upstream $\left(\sim 156\right.$ Earth radii, $\left.R_{E}\right)$ from the shock by ion beam instability, are cascading to smaller or larger scales, depending on the models. Far upstream, wave-particle interactions have been observed up to more than $80 R_{E}$ distance from the shock, with both Alfvénic and magnetosonic fluctuations generated far upstream (Tsurutani and Rodriguez, 1981; Sanderson et al., 1985; Meziane et al., 1997, 2001). In our models we assume that both right- and left-handed Alfvénic fluctuations are generated.

The mechanism of energy transfer between MHD waves is still controversial. From the observational viewpoint, the process seems to be the regular cascade, whereas from the numerical and the theoretical viewpoint within a onedimensional system, the process is found to be the inverse cascade. The monochromatic ULF waves with longer than $30 \mathrm{~s}$ period were observed at more than several $R_{E}$ distance from the bow shock (Eastwood et al., 2005), whereas short duration $(5-20 \mathrm{~s})$ structures in SLAMS were observed near the bow shock (Wilkinson, 2003). Therefore, if wave-wave interaction occurs between these ULF waves, their frequencies become higher while approaching the shock, i.e. the regular cascade takes place. On the other hand, in the large-scale hybrid simulations at a parallel shock done by Giacalone (2004), the high frequency monochromatic waves are selfexcited by accelerated particles far upstream, and the lower frequency waves are generated while approaching the shock. Accordingly, the turbulent field with a power-law spectrum is generated near the shock. Hence, if there are wave-wave interactions between the waves approaching the shock, the inverse cascade will take place. Moreover, according to a standard schema of the parametric decay instability of Alfvén waves in a one-dimensional system, a parent wave decays into a daughter wave with a frequency lower than the parent wave frequency; inverse cascading takes place. However, at a nonlinear stage, energy exchange exists in both directions between parent and daughter waves (Nariyuki and Hada, 2006). Therefore, both cascading models used here are consistent with the observations and the simulations in the upstream of a parallel shock.

It is an observational fact for quasi-parallel interplanetary shocks that the wave energy increases toward the shock by gaining from energetic particles through wave-particle interaction (Kennel et al., 1984a,b; Sanderson et al., 1985). However, in the simulation of Giacalone (2004), the increase in wave energy toward the shock cannot be described only by wave-particle interactions, and the importance of a nonlinear evolution of the waves such as the SLAMS are suggested as explaination of the excess of the observed wave energy near 
the shock. Further, at the foreshock region of the Earth's bow shock, Hada et al. (2003a) have revealed that the wave phase correlation, which characterizes the nonlinear wavewave interaction, becomes stronger as the MHD wave amplitude becomes larger. Therefore, not only wave-particle interaction but also wave-wave interaction should be taken into account as a generation mechanisms of the upstream turbulence. In our wave cascade models, spatial evolution of the wave power spectrum causes increased turbulence energy toward the shock. In this paper we focus on the spatial dependence between the particle mean free path $\lambda_{\|}$and the modeled turbulence.

The spatially dependent $\lambda_{\|}$in the upstream of a parallel shock causes modification of the DSA process. The results of Giacalone (2004) imply that the mean free path of accelerated particles increases with distance in the upstream of the shock. Also, spatial profile of accelerated particles becomes flat far upstream due to the presence of escaping particles, since there is no sufficient turbulence to scatter them. In the discussion section, we compare our results with those of Giacalone (2004).

\section{Numerical model}

\subsection{Magnetic field fluctuations}

Let us consider a region in the upstream of a parallel shock, and assume a one-dimensional system with $x$ axis as the spatial coordinate, where the background magnetic field and the solar wind velocity $u$ are parallel to the $x$ axis. The mean velocities of the energetic particles are assumed to be equal to the solar wind velocity $u$. Hence, the typical particle velocity far exceeds the Alfvén wave velocity $v_{A}$, we let the waves be stationary and convected by the solar wind. In this paper, we employ three different models of one-dimensional MHD turbulence: regular cascade, inverse cascade, and homogeneous turbulence models. In all three models, the fluctuations in the transverse fields are written as

$\delta B_{y}+i \delta B_{z}=\sum_{k} \delta B_{k}(x) \exp \left[i k(x-u t)+i \phi_{k}\right]$,

where positive and negative wavenumbers $k$ correspond to right- and left-handed polarizations, respectively, and phases $\phi_{k}$ are random constants. The $y$ and $z$ axes form an orthogonal right-handed system. The normalizations $v_{A} / \Omega$ and $\Omega$, respectively for space and time, are used, where $\Omega$ is the proton gyro-frequency for an averaged magnetic field $B_{0}$. Here we choose the typical solar wind parameters: $v_{A}=50 \mathrm{~km} / \mathrm{s}$ and $\Omega=1 / \mathrm{s}$. Different plasma flow velocities are used as $u=100 \mathrm{~km} / \mathrm{s}, u=400 \mathrm{~km} / \mathrm{s}$, and $u=1600 \mathrm{~km} / \mathrm{s}$, i.e., $u=2 v_{A}, u=8 v_{A}$, and $u=32 v_{A}$, respectively. In total, 125 wave mode numbers for each right- and left-handed polarizations are introduced in the system. The mode numbers are in the range of $4 \leq m \leq 128$, where wavenumbers are given by $k=2 \pi m / 1024$. Accordingly, the wave lengths are in the range from 0.0625 to $2 R_{E}$. The particle motion in the simulation is not so sensitive to the wave length for wave modes higher than $m=128$, as discussed in the result section 3.3.2. The maximum wave length, $2 R_{E}$, is about twice the wave length of the quasi-monochromatic fast magnetosonic waves observed in the foreshock region (Eastwood et al., 2005). We assume that the position near $x=0$ is the wave source, and that shock position is $x=x_{s h}=20480 v_{A} / \Omega$, which corresponds to $156 R_{E}$. The periodic turbulence exists for $x>x_{s h}$. The spatial size of the foreshock region, $156 R_{E}$, is almost a half of the distance of a free-escape boundary from the shock in the hybrid simulations of Giacalone (2004).

\subsubsection{Amplification of magnetic field fluctuations}

An important feature of our fluctuation field models is that the amplitude of each Fourier mode is spatially amplified. The spatial amplifications are different in the three models. For homogeneous turbulence model, the amplitude is constant, i.e. $\delta B_{k}(x)=\delta B_{k}$, and the power spectrum $P_{k}$ is powerlaw type with an index $\gamma$, namely $P_{k} \sim|k|^{-\gamma}$. On the other hand, for the other two cascade models, $\delta B_{k}(x)$ is written as

$\delta B_{k}(x)=\sqrt{P_{k}}\left(\frac{1}{\pi} \tan ^{-1}\left[\frac{x-x_{0}^{k}}{\Delta x}\right]+\frac{1}{2}\right)$.

The function in the parentheses goes to zero for $x \ll x_{0}^{k}$, goes to unity for $x \gg x_{0}^{k}$, and maximally grows at around $x=x_{0}^{k}$ with its width $\Delta x$. Thus the growth rate corresponds to $u / \Delta x$, and typically we choose the growth width $\Delta x=256$ in the unit length, corresponding to $\Delta x=2 R_{E}$. The locations of the wave excitation $x_{0}^{k}$, as a function of wavenumber $k$, depend on a model. We divide the 125 mode numbers into 18 groups, and the excitation position for the $j$ th group is given as $x_{0}^{k}=512+(j-1) 1024$. In the regular cascade model, waves with lower wavenumbers are assigned to the smaller $\mathrm{j}$-th group in the far upstream region. In the inverse cascade model, waves with higher wavenumbers are assigned to the smaller $\mathrm{j}$-th group. We assume the waves in the first group are excited by wave-particle interaction, whereas the waves in the other groups approaching the shock are generated by wave-wave interaction.

\subsubsection{Wave source}

We describe the wave generation scheme in the first group. In the upstream of a parallel shock, Alfvén waves, which propagate in the same direction of reflected ion beam at the shock front, are excited by ion beam instability. The generated wave energy is comparable to the beam particle energy. For the regular cascade model, the wave with $k=0.0245(m=1)$ and $\delta B^{2} / B_{0}^{2}=0.14$ is assumed to be generated far upstream. Whereas for the inverse cascade model, the waves with $0.626 \leq k \leq 0.785(102 \leq m \leq 128)$ and $\delta B^{2} / B_{0}^{2}=0.025$ are assumed. Here the wave energy $\delta B^{2}$ is defined by summa- 
(A)

(B)

(C)

(a)

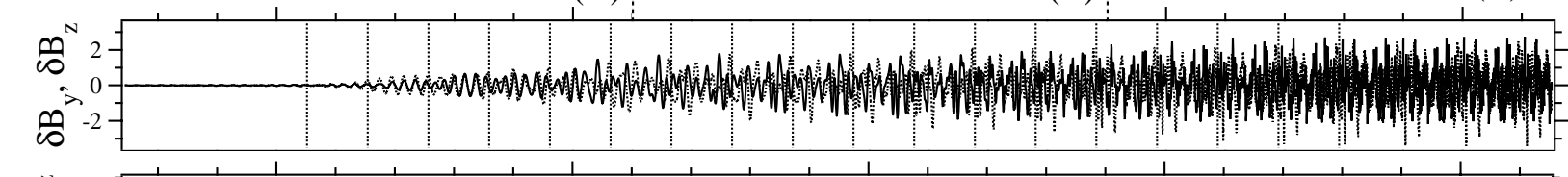

(b)

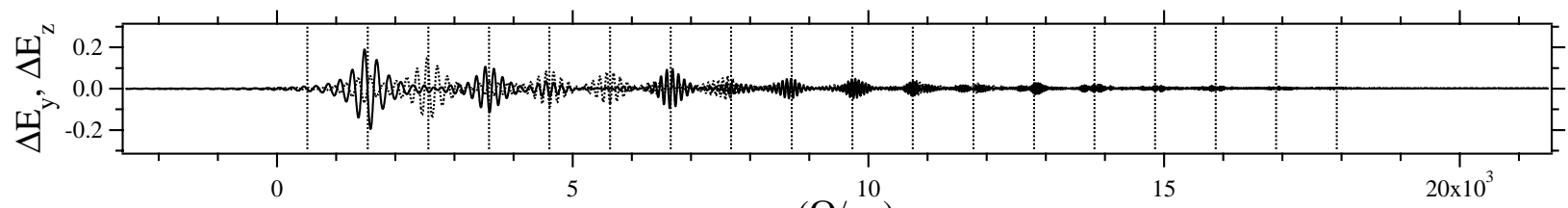

(A)

(B)

(C)

(c)

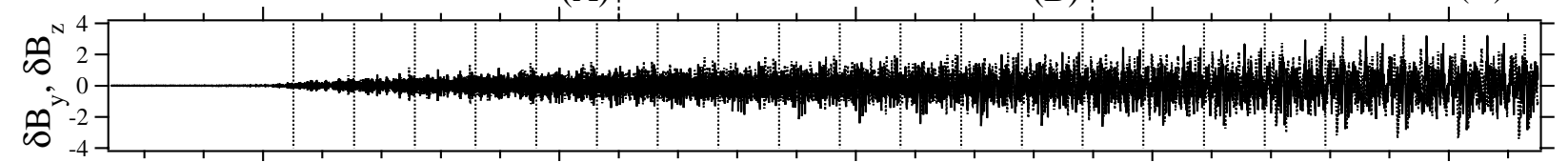

(d)

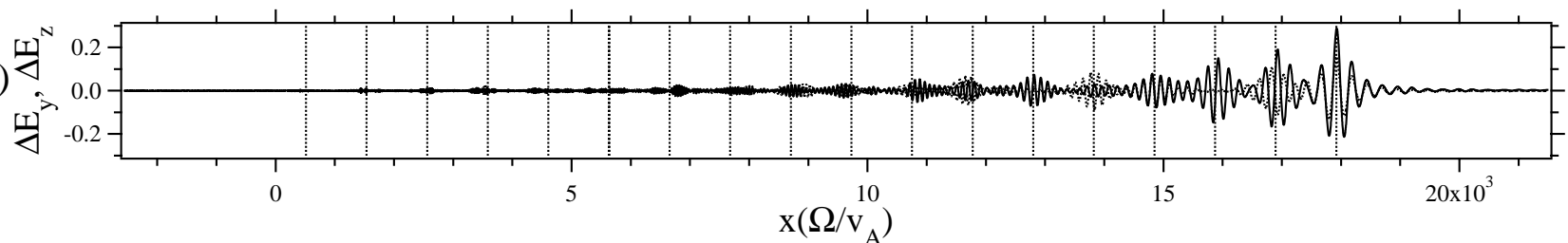

Fig. 2. Two cascade models. Magnetic field fluctuations are shown in (a) and (c) for the regular and inverse cascade models, respectively. Electric field fluctuations, with the motional electric field extracted, are shown in (b) and (d) for the regular and inverse cascade models, respectively. At the regions labelled by (A), (B), and (C), the power spectra of the magnetic field fluctuations are shown in Fig. 1 with the same labellings.

tion of $P_{k}$ for the waves in the first group. Now we find the beam velocity $v_{b}$ and beam density $n_{b}$, corresponding to the generated waves. By combination of the cyclotron resonance condition $\omega-k v_{b}=-\Omega$ and the dispersion relation of Alfvén waves $\omega=k v_{A}$, we obtain $v_{b}=v_{A}+\Omega / k$. The ratio of the beam density $n_{b}$ to the background plasma density $n_{0}$ is $n_{b} / n_{0}=\left(v_{A} / v_{b}\right)^{2}\left(\delta B / B_{0}\right)^{2}$, where the equality between beam particle energy and wave energy is assumed. Then, the parameters are calculated as $v_{b} / v_{A}=42$ and $n_{b} / n_{0}=8.3 \times 10^{-5}$ for the regular cascade model, and $v_{b} / v_{A}=2.4$ and $n_{b} / n_{0}=0.012$ for the inverse cascade model.

\subsubsection{Wave cascade models}

The wave generation in the other groups with $j \geq 2$ is assumed to be wave cascading, as the spectrum shape is defined by the spectral index $\gamma$. As one approaches the shock, the waves in each group are generated, so that the increments of the total wave energy, which are about 0.025 , are the same in the two cascade models. Figure 2a shows the magnetic field fluctuations for the regular cascade model. The solid and dotted lines show two components $\delta B_{y}$ and $\delta B_{z}$, respectively. The vertical dotted lines show positions $x_{0}^{k}$ for 18 groups, where the waves in each group are generated. The developed power spectra in the regions (A), (B), and (C) cor- respond to different locations for the regular cascade model shown in Fig. 1. In the region (A) up to the length $x=6000$, five mode numbers from the lowest wavenumber are excited with their amplitudes $\sim \sqrt{P_{k}}$, and other modes are not generated. Hence, taking the sum of all these modes in Eq. (1), the quasi-monochromatic waves are generated in the region (A). The monochromatic fluctuations in the region (A) excite fluctuations in the region (C). Figure $2 \mathrm{c}$ shows magnetic field fluctuations for the inverse cascade model, and the notations are the same as in the Fig. 2(a). In the region (A), 95 mode numbers from the highest wavenumber are excited and the waves with high wavenumbers are generated. In the region (C) the fluctuations in both models are statistically the same, and the turbulence level $\delta B / B_{0}=1$ for right- and left-handed polarizations are also the same with those in the homogeneous turbulence model.

\subsection{Electric field fluctuations}

For the given magnetic field fluctuations, the electric field fluctuations are obtained by numerical integration of Faraday's law,

$$
E_{y}+i E_{z}=\frac{u}{c} \sum_{k} \exp \left[-i k u t+i \phi_{k}\right] \int \delta B_{k}(x) e^{i k x} d x,
$$


where the spatial integration is performed by the Simpson method. If $\delta B_{k} \sim$ const as in the homogeneous turbulence model, the frozen-in condition is satisfied as $\mathbf{E}=-\mathbf{u} \times \mathbf{B} / c \equiv$ $\mathbf{E}_{f r}$, and within this case particle energy is conserved in the plasma rest frame. However, in the two cascade models an additional electric field other than the frozen-in field or motional electric field $\mathbf{E}_{f r}$ appears due to amplification of $\delta B_{k}$. The particle energy is not conserved in the plasma rest frame anymore. The additional electric fields are not important for the parallel diffusion, but important for particle acceleration.

Figures $2 \mathrm{~b}$ and $2 \mathrm{~d}$ show the additional electric field $\Delta \mathbf{E}=\mathbf{E}-\mathbf{E}_{f r}$, with the two components $\Delta E_{y}$ and $\Delta E_{z}$, for the regular and the inverse cascade models, respectively. The additional electric field appears with a packet-like shape at excitation position $x_{0}^{k}$. In the regular cascade model, the magnitude of $\Delta \mathbf{E}$ is largest far upstream, and then decreases toward the shock. On the contrary in the inverse cascade model, the magnitude is largest near the shock, and then decreases away from the shock. This indicates that the waves with lower wavenumbers produce larger additional electric fields. We also make a remark on the amplitude of $\Delta \mathbf{E}$. The additional electric field disappears far from $x_{0}^{k}$, when growth width $\Delta x$ is comparable or larger than the wave length $l$. However, $\Delta \mathbf{E}$ remains far from $x_{0}^{k}$ when $\Delta x<l$, i.e., rapid amplification of the wave is assumed. Thus acceleration due to $\Delta \mathbf{E}$ will be remarkable for $\Delta x<l$, although this case is not used in the present work.

\subsection{Statistics of particle motions}

In the given electromagnetic field, we integrate the ion equation of motion over time by the Buneman-Boris method,

$m \frac{d \mathbf{v}}{d t}=q\left(\mathbf{E}+\frac{\mathbf{v}}{c} \times \mathbf{B}\right) ; \quad \frac{d x}{d t}=v_{x}$

where the electric field fluctuations $\mathbf{E}=\left(0, E_{y}(x, t), E_{z}(x, t)\right)$ are given by Eq. (3) and the magnetic field $\mathbf{B}=\left(B_{0}, \delta B_{y}(x, t), \delta B_{z}(x, t)\right)$ is introduced in Eq. (1). We assume the test particles are the solar wind thermal particles rather than accelerated particles. Hence, the initial particle positions are far upstream at $x_{0}=3000$ and the initial velocities are given by the Maxwellian distribution shifted by the plasma flow velocity $u$

$f(\mathbf{v})=\frac{1}{\pi^{3 / 2} v_{t h}^{3}} \exp \left[-\frac{(\mathbf{v}-u \hat{x})^{2}}{v_{t h}^{2}}\right]$

where the initial thermal velocity is $v_{t h}$ and the initial proton temperature is defined by $E_{0}=3 m v_{t h}^{2} / 2$. The thermal velocity $v_{t h}=400 \mathrm{~km} / \mathrm{s}$ corresponds to $\sim 2.5 \mathrm{keV}$. For the fixed plasma flow $u$ and for each of the fluctuation models, we calculate 11 runs with different $v_{t h}$ corresponding to the temperature range from $0.157 \mathrm{keV}$ to $160 \mathrm{keV}$.
To estimate the spatial dependence of the mean free path, we evaluate the diffusion coefficient, keeping the time scale dependence,

$D_{\|}(t)=\frac{<(x-\bar{x})^{2}>}{t}$,

where $x$ is a particle position for a time scale $t$, and $\bar{x}$ is the mean position defined by $\bar{x}=x_{0}+u t$, and $<>$ denotes an ensemble average of $N$ particles. For the normal diffusion, $D_{\|}$ is independent of time. However, in general, $D_{\|}$is expected to be a function of space and time $D_{\|}(x, t)$. Here we assume the spatial coordinate $x$, being in a co-moving frame with the solar wind $x=x_{0}+u t \equiv x_{s w}$, where the subscript 'sw' represents the solar wind rest frame. Then, the spatial dependence of $D_{\|}$is evaluated by the transformation of Eq. (6) from time to space as

$D_{\|}(t)=D_{\|}\left(\frac{x-x_{0}}{u}\right)$.

Finally, we obtain the spatially dependent mean free path as $\lambda_{\|}(x)=3 D_{\|}(x) / v_{t h}$. The estimation of the spatially dependent diffusion shown here is possible only when the convection by the solar wind exists. In general, however, the dependence of $D_{\|}$on $x$ and $t$ should be determined independently.

In addition, we discuss dependence of the acceleration process on the additional electric field due to spatially amplified magnetic field. The variance of velocity is evaluated by,

$\sigma_{v v}(t)=<\left(v_{x}-u\right)^{2}>+<v_{y}^{2}>+<v_{z}^{2}>$,

where the mean velocity in the $x$ direction is assumed equal to $u$, and in the $y$ and the $z$ directions are zero. These assumptions are valid for the time scale we consider in this paper (see Sect. 3.3). We calculate at least two sets of random phases $\phi_{k}$ of the fluctuations in Eq. (1) for a single run. For a single set of random phases $\phi_{k}$, i.e. single field pattern, 256 particles are traced. In the two cascade models, some particles escape far upstream, and these escaping particles are not used for the ensemble average of $N$ particles in Eqs. (6) and (8). In that case, several field patterns are used to avoid decreasing in the number of the particles, so that the number of particles is at least $N>250$.

\subsection{Spatially dependent $\lambda_{\|}$based on the QLT}

In the QLT, the parallel diffusion coefficient $D_{\|}^{Q L}$ is estimated by the integration of the pitch angle diffusion coefficient for the wave power spectrum index in the range of $1<\gamma<2$ (Schlickeiser, 2002). Then the mean free path $\lambda_{\|}^{Q L}$ is obtained from $D_{\|}^{Q L}=v_{t h} \lambda_{\|}^{Q L} / 3$ as

$\lambda_{\|}^{Q L} \simeq\left(\frac{B_{0}}{\delta B}\right)^{2}\left(\frac{\Omega}{k_{\min } v_{t h}}\right)^{\gamma-1} \frac{3 v_{t h}}{\Omega}$

where the background magnetic field $B_{0}$, fluctuation amplitude $\delta B$, minimum wavenumber $k_{\min }$, gyro-frequency $\Omega$, are 
(a)

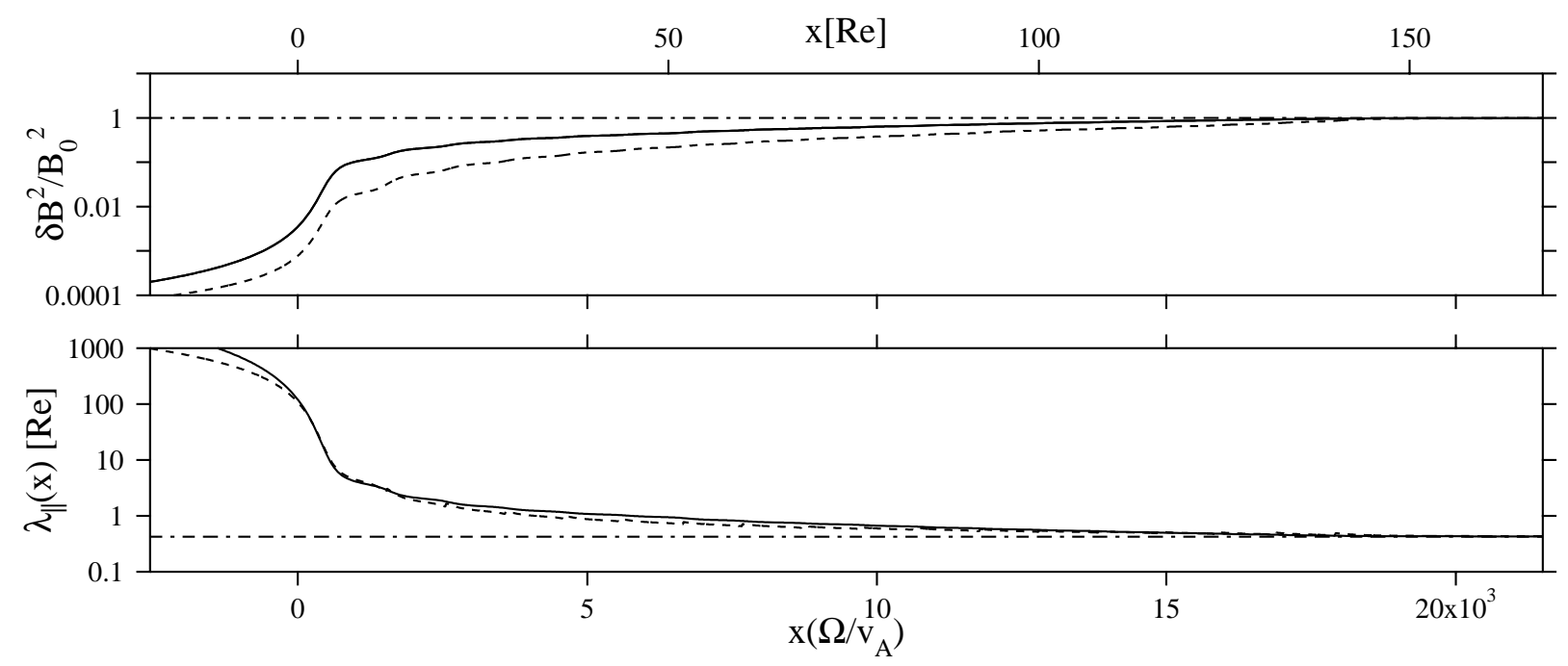

Fig. 3. (a) Magnetic field fluctuation intensity and (b) the spatially dependent mean free paths based on the QLT(quasi-linear theory) in Eq. (9) and Eq. (10). Dotted-dashed, solid, and dashed lines are for the homogeneous turbulence, the regular cascade, and the inverse cascade models, respectively.

introduced. Hence, the velocity dependence is $\lambda_{\|}^{Q L} \sim v_{t h}^{2-\gamma}$. For the homogeneous turbulence model, the turbulence en$\operatorname{ergy} \delta B^{2}=1$ and $\lambda_{\|}^{Q L}$ in Eq. (9) are shown in Fig. 3a and b by dotted-dashed lines, respectively.

On the other hand, for two cascade models, the spatial dependence appears through a spatial variations of the MHD turbulence. We assume in Eq. (9) the spatial variations of the MHD turbulence, and then obtain the spatially dependent mean free path $\lambda_{\|}^{Q L}$ based on the QLT as

$\lambda_{\|}^{Q L}(x) \simeq\left(\frac{B_{0}}{\delta B(x)}\right)^{2}\left(\frac{\Omega}{k_{\min }(x) v_{t h}}\right)^{\gamma-1} \frac{3 v_{t h}}{\Omega}$.

In this paper, $\delta B^{2}$ varies for both cascade models, whereas $k_{\min }$ varies only for the inverse cascade model. Figure 3a shows the spatial variations of $\delta B^{2}(x)$, with solid and dashed lines for the regular and the inverse cascade models, respectively. The turbulence energies are obtained as $\delta B^{2}(x)=\sum_{k} \delta B_{k}^{2}(x)$, by using Eq. (2) where the summation was taken in the right- and the left-handed polarizations separately. For both cascade models, $\delta B^{2}(x)$ increases toward the shock, and is saturated at $\delta B^{2}=1$ for $x \sim 20000 v_{A} / \Omega \sim 156$ $R_{E}$. The spatial variations of the two cascade models are not exactly the same. Figure $3 \mathrm{~b}$ shows $\lambda_{\|}^{Q L}(x)$ in Eq. (10) with solid and dashed lines for the regular and the inverse cascade models, respectively. If $\delta B^{2}(x)$ is only considered, $\lambda_{\|}^{Q L}(x)$ for the inverse cascade model should be smaller than $\lambda_{\|}^{Q L}(x)$ for the inverse cascade model. However, spatial variation of $k_{\min }(x)$ for the inverse cascade model leads that the $\lambda_{\|}^{Q L}(x)$ for two cascade models are almost equal. We compare the numerical $\lambda_{\|}(x)$ from Eq. (7) with the theoretical $\lambda_{\|}^{Q L}$ based on the QLT in Eq. (10) for two cascade models.

\section{Results}

\subsection{Single particle motion}

First we discuss single particle motion for three different MHD turbulence models. In general, nonlinear motion of a single particle in a one-dimensional space has been analyzed based on the Hamiltonian for the particle motion (e.g., Kuramitsu and Krasnoselskikh, 2005). In the presence of finite amplitude MHD wave with a single mode, the corresponding Hamiltonian can be described as a time-independent function in phase space coordinates which are pitch angle cosine in the wave rest frame $\mu$ and phase $\psi$ between transverse magnetic field fluctuation and transverse velocity vectors. The particle trajectory in the $\psi-\mu$ space follows the contour lines of the Hamiltonian $H(\psi, \mu)$ for a single wave. When the wave amplitude is finite, the closed orbits appears in the $\psi-\mu$ space, representing trapping by the wave with a velocity around resonance velocity, $k v_{\|}=-\Omega$, where $k$ is a wavenumber of a single wave and $v_{\|}$is parallel velocity in the wave (plasma) rest frame. In our model, the phase space trajectory deviates from the contour lines of $H(\psi, \mu)$ due to two effects, i.e., the spatial variation of the wave amplitude and the presence of many wave modes.

Figure 4 shows particle position $x-u t$ (left panels), velocity space (middle panels), and phase space (right panels) trajectories in the plasma rest frame for a single particle in three different turbulence models. In the phase space each 

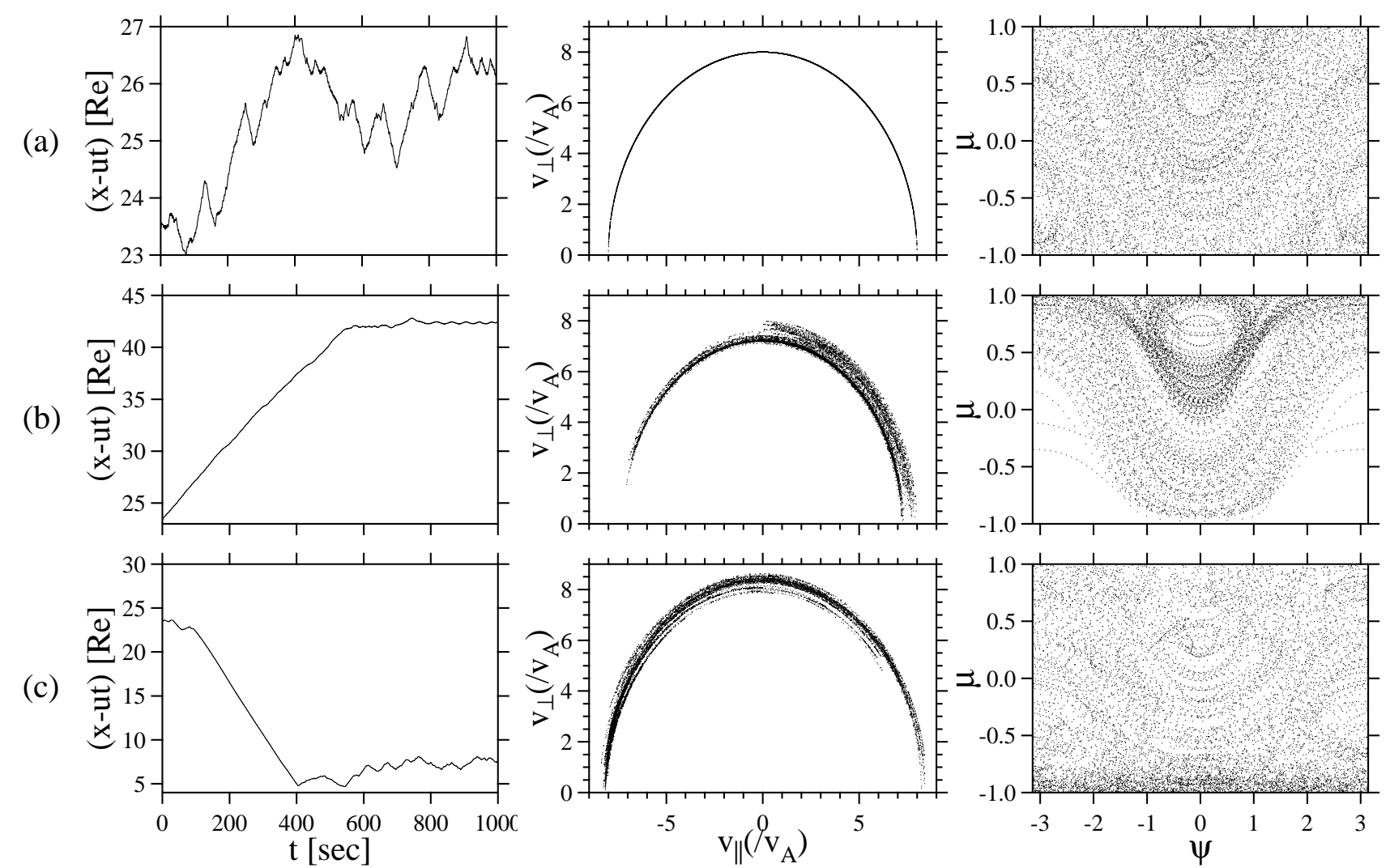

Fig. 4. Particle position (right panels), velocity space trajectories (middle panels), and phase space trajectories (right panels) in the plasma flow rest frame for a single particle motion for (a) homogeneous, (b) regular cascade, and (c) inverse cascade turbulence models. The parameters used are $u=8, v=8$, where $v$ represents magnitude of initial velocity of the particle in the plasma rest frame.

dot represents a trajectory of a single particle at each time step. The parameters are $u=8$ and $v=8$, where the magnitude of the initial velocity of the particle in the plasma rest frame is $v=\sqrt{\left(v_{x}-u\right)^{2}+v_{y}^{2}+v_{z}^{2}}$. In the velocity space, the parallel and perpendicular velocities to the background magnetic field are $v_{\|}=v_{x}-u$ and $v_{\perp}=\sqrt{v_{y}^{2}+v_{z}^{2}}$, respectively. The phase space coordinates $\mu$ and $\psi$ are defined by,

$\mu=v_{\|} / v ; \quad \sin \psi=\frac{(\mathbf{v} \times \boldsymbol{B})_{x}}{v_{\perp} \delta B_{\perp}}$.

Here the transverse fluctuation magnitude is $\delta B_{\perp}=\sqrt{\delta B_{y}^{2}+\delta B_{z}^{2}}$. The velocities are obtained numerically from Eq. (4), and the particle motion is affected by the fluctuations at each time step. Let us note the definition of the phase $\psi$. The $\psi$ in general is defined for a single mode, whereas in Eq. (11) it is defined for local wave modes, and the modes interacting with the particle changes in time.

First, let us look at the homogeneous turbulence model (Fig. 4a). In the $x-u t$ space the orbit looks Brownian, in the velocity space the scattering is isotropic, and in the $\psi-\mu$ space the trajectory covers the whole phase space region. We find that the pitch angle scattering occurs effectively and isotropically, and that particles frequently traverse the 90 degree pitch angle, i.e. $\mu=0$. The frequent traverse across $\mu=0$ corresponds the frequent reversal of the parallel velocity. Consequently, the particles are traveling back and forth along the mean field, resulting in the Brownian motion in the $x-u t$ space. Also, the Brownian motion represents that the sign of $\mu$ is randomly reversed. Resonance broadening, which is the consequence of the finite amplitude waves, causes the pitch angle diffusion over $90^{\circ}$ (Hada et al., 2003b), since the wave amplitude is large, i.e. $\delta B / B_{0}=1$.

For the regular cascade model (Fig. 4b), the trajectories are composed of mainly two parts. In the $x-u t$ space, the particle travels almost with a constant velocity along the mean field ('walk') up to $500 \mathrm{sec}$, and at later time stays almost at the same position for a long time ("sticking"). In the $\psi-\mu$ space, the trajectory is trapped around certain positive $\mu$, and at other times the trajectory is open along the $\psi$ coordinate and is periodically across $\mu=0$. The former represents trapping by a wave with low wavenumber far upstream. The 


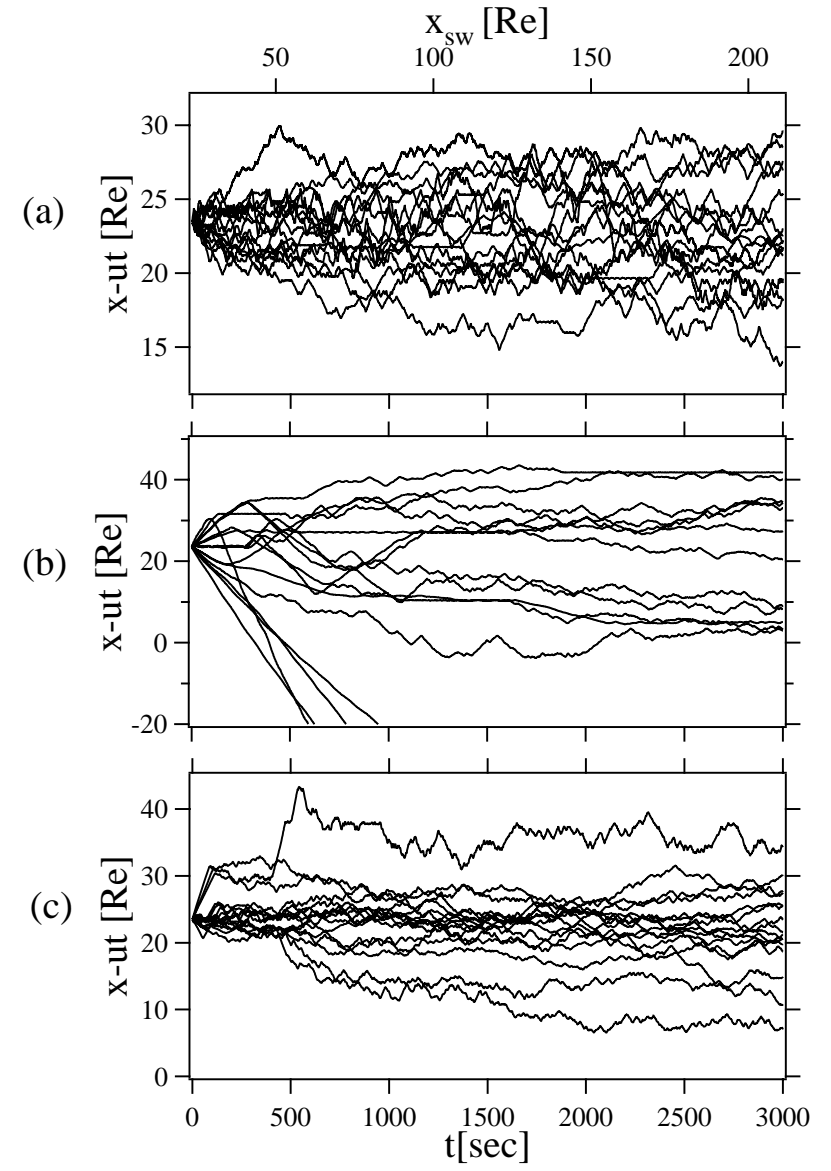

Fig. 5. Particle positions in the plasma flow rest frame $x-u t$ as a function of time $t$ and space $x_{s w}=x_{0}+u t$ in co-moving frame with the plasma flow, shown in bottom and top axes, respectively, for (a) homogeneous (b) regular cascade, and (c) inverse cascade turbulence models. The parameters used are $u=8, v_{t h}=8$.

certain positive $\mu$ corresponds to resonance with the wave. The trapped trajectory in the $\psi-\mu$ space corresponds to the "walk" segment in the $x-u t$ space, with the almost constant velocity being equal to the resonance velocity on average. On the other hand the open trajectory in the $\psi-\mu$ space represents that the parallel velocity is almost zero on average, and thereby corresponds to the "sticking" segment in the $x-u t$ space. As a consequence of the coherent pitch angle scattering such as the trapping by a wave with low wavenumber, the particle motion in the $x-u t$ space appears to be nonBrownian motion, which is composed of "walk" and "sticking" type motions. The non-Brownian motion reflects that the pitch angle diffusion over $90^{\circ}$ is not random.

For the inverse cascade model (Fig. 4c), in the $x-u t$ space there is also "walk" motion up to $400 \mathrm{~s}$, and Brownian like orbit at a later time. In the $\psi-\mu$ space, the trajectory is sometimes concentrated around $\mu \sim-1$, and other times it

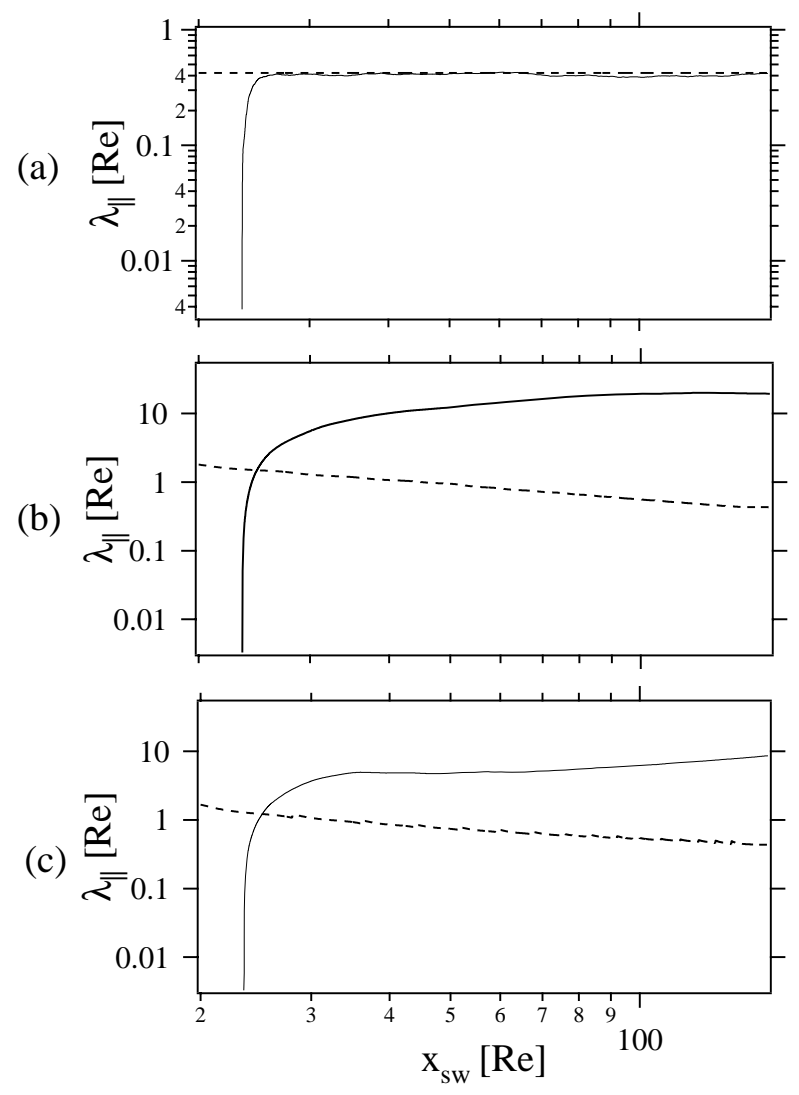

Fig. 6. Mean free path $\lambda_{\|}$as a function of space in co-moving frame with the plasma flow for (a) homogeneous (b) regular cascade, and (c) inverse cascade turbulence models. The parameters used are the same as in Fig. 5. Solid and dashed lines show numerical $\lambda_{\|}$and theoretical values in Eqs. $(9,10)$, respectively.

covers the whole phase space region. The former represents the anisotropic pitch angle scattering, corresponding to the walk motion in the $x-u t$ space. Here we refer to a test particle simulation done by Hada et al. (2003b). In the standard QLT, the pitch angle diffusion is absent at $|\mu|=1$ due to force free for the particle motion along the mean field. When $\delta B / B_{0}=1$ the standard QLT fails, and the pitch angle diffusion rate around $|\mu|=1$ is finite but still small compared with those at other pitch angles. Therefore, we think that the weak scattering around $|\mu|=1$ causes the anisotropic pitch angle scattering, resulting in the walk motion in the $x-u t$ space.

\subsection{Spatial dependence of the mean free path}

Next we discuss statistics of particle motion along field lines. Figure 5 shows several particle positions in the plasma flow rest frame $x-u t$ as functions of time $t$ and space $x_{s w}=x_{0}+u t$, in co-moving frame with the plasma flow, for 
(a) the homogeneous turbulence, (b) the regular cascade, and (c) the inverse cascade models. The plasma flow and the thermal velocities for all runs are $u=8$ and $v_{t h}=8$, respectively. Figure 6 shows the mean free path $\lambda_{\|}$, as a function of space $x_{s w}$. In each panel, the calculated $\lambda_{\|}$is the same run as in each panel of the Fig. 5, and the solid and dashed lines represent the numerical $\lambda_{\|}$and the theoretical values in Eqs. (9, $10)$, respectively.

In the homogeneous turbulence model, the orbits look more or less similar to the Brownian orbits (Fig. 5a), and the mean free path reaches a constant value (Fig. 6a), thus representing normal diffusion. We find that the numerical $\lambda_{\|}$ matches well with the quasi-linear formula $\lambda_{\|}^{Q L}$ in Eq. (9). Therefore, the QLT for the parallel diffusion is valid for the homogeneous turbulence model.

In the regular cascade model, the orbits look quite different from those in the homogeneous turbulence case. In Fig. 5b, most of the particles are traveling along the mean field within the short time scale $t<500$ s ("walk" motion), and some of the particles are escaping far upstream (shown by out of the minimum scale in vertical axis of the figure). The "walk" motion corresponds to particles being in resonance with waves with low wavenumbers as discussed in Sect. 3.1. The escaping particles are non-resonant particles. In the far upstream region $x<6000$, there are only waves with low wavenumbers in the range of $0.025 \leq|k| \leq 0.05$, and corresponding the resonance velocity is $20 \leq v_{\|} \leq 40$, where the parallel velocity in the linear resonance condition is assumed . Therefore, non-resonant particles exist in the center of the Maxwellian distribution since the thermal velocity is $v_{t h}=8$, and these particles can be the escaping particles. In Fig. 6b, The calculated $\lambda_{\|}$is much increased than $\lambda_{\|}^{Q L}$ in the far upstream region $\left(x<50 R_{E}\right)$. At the shock, the numerical value is about $\lambda_{\|}=20 R_{E}$, compared with $\lambda_{\|}^{Q L}=0.4 R_{E}$. We also find the discrepancy of spatial dependence of $\lambda_{\|}$between the simulation and prediction based on the QLT. Namely, the calculated $\lambda_{\|}$gradually increases toward the shock, whereas $\lambda_{\|}^{Q L}(x)$ decreases toward the shock. The increased $\lambda_{\|}$is due to the walk motion of the resonant particles. This discrepancy reveals that the spatial dependence of $\lambda_{\|}$is not only described by the spatial evolution of turbulence energy $\delta B^{2}(x)$.

In the inverse cascade model, a few particles have positive "walk" segment at $t<500 \mathrm{~s}$, corresponding to $x_{s w}<50 R_{E}$. Trajectories of other particles look like the Brownian orbits (Fig. 5c). The former is probably due to two reasons. One is due to the anisotropic pitch angle diffusion for finite time scale as discussed at Sect. 3.1. The other is due to the presence of non-resonant particles. Far upstream $x<6000$, there are only waves with high wavenumbers $0.208 \leq|k| \leq 0.785$, and corresponding resonance velocity is $1.27 \leq v_{\|} \leq 4.8$ where the parallel velocity in the linear resonance condition is assumed. Therefore, the particles, in the tail of the Maxwellian distribution with the range of $v_{\|}>4.8$, cannot resonate with the waves, and sometimes they travel ballistically toward the shock with their own velocities. The numerical $\lambda_{\|}(x)$ is dominantly increased in the far upstream region and is gradually increased toward the shock. This is due to the anisotropically scattered particles or the non-resonant particles. At the shock the numerical value is about $\lambda_{\|}=10 R_{E}$ (Fig. 6c). The spatial dependence of the calculated $\lambda_{\|}$is also inconsistent with the $\lambda_{\|}^{Q L}$.

\subsection{Mean Free Path at the Shock}

Figure 7 summarizes the results of the evaluated mean free path $\lambda_{\|}$at the shock front, $x=20600$ in the unit length, corresponding to $x \sim 161 R_{E}$. The upper (a) and lower (b) panels correspond to the regular and the inverse cascade models, respectively. In each panel, the symbols represent the numerical $\lambda_{\|}$for different plasma flow velocities, $u=2(0)$, $u=8(\triangle)$, and $u=32(\square)$. Also, the numerical $\lambda_{\|}$for the homogeneous turbulence model at $u=8$ is shown by the symbols (+) in each panel. The solid line represents the $\lambda_{\|}^{Q L}$ of the quasi-linear theory in Eq. (9), and the dependence on $v_{t h}$ is $\lambda_{\|}^{Q L} \sim v_{t h}^{0.5}$ for $\gamma=1.5$. The evaluated $\lambda_{\|}$are plotted as functions of thermal velocity $v_{t h}$ and the initial thermal temperature $E_{0}=3 m v_{t h}^{2} / 2$ in $\mathrm{keV}$, in the bottom and upper axes, respectively. The evaluation time $T$ depends on $u$, since the evaluation position $x$ is fixed as $x=x_{0}+u T=20600$. The evaluation time corresponds to the convection time sweeping from the monochromatic waves far upstream to the turbulent waves at the shock. The convection time to the shock front becomes shorter, as the plasma flow becomes faster. The evaluation times are $T=8800,2200$, and 550 for $u=2,8$, and 32 , respectively.

\subsubsection{Resonance velocity in the QLT}

Particles interact with waves only if resonance conditions are satisfied. We assume that $v_{t h}$ is equal to the parallel velocity of a particle in the linear resonance condition. Then, we obtain the minimum and maximum thermal velocities $v_{\min }$ and $v_{\max }$ to resonate with the waves in the system, shown with vertical dashed lines in each panel. The values are $v_{\min }=\Omega / k_{\max }$ and $v_{\max }=\Omega / k_{\min }$. Hence, $v_{\max }$ becomes larger if longer wave length is introduced into the system. The waves resonate with the particles in the velocity range of $v_{\min } \leq v_{t h} \leq v_{\max }$. In the range of $v_{t h}>v_{\max }$, the numerical $\lambda_{\|}$in all runs are much enhanced, sometimes the values of $\lambda_{\|}$are out of the system size, $\sim 161 R_{E}$. For this case most of the particles do not interact with the waves, and propagate ballistically with their own initial velocities. Therefore, the diffusion coefficient increases with elapsed time, and the $\lambda_{\|}$ becomes large.

\subsubsection{Homogeneous turbulence model}

Let us look at the region with $v_{t h}<v_{\max }$. In the homogeneous turbulence model, the numerical $\lambda_{\|}$matches well $\lambda_{\|}^{Q L}$ except 
(a)

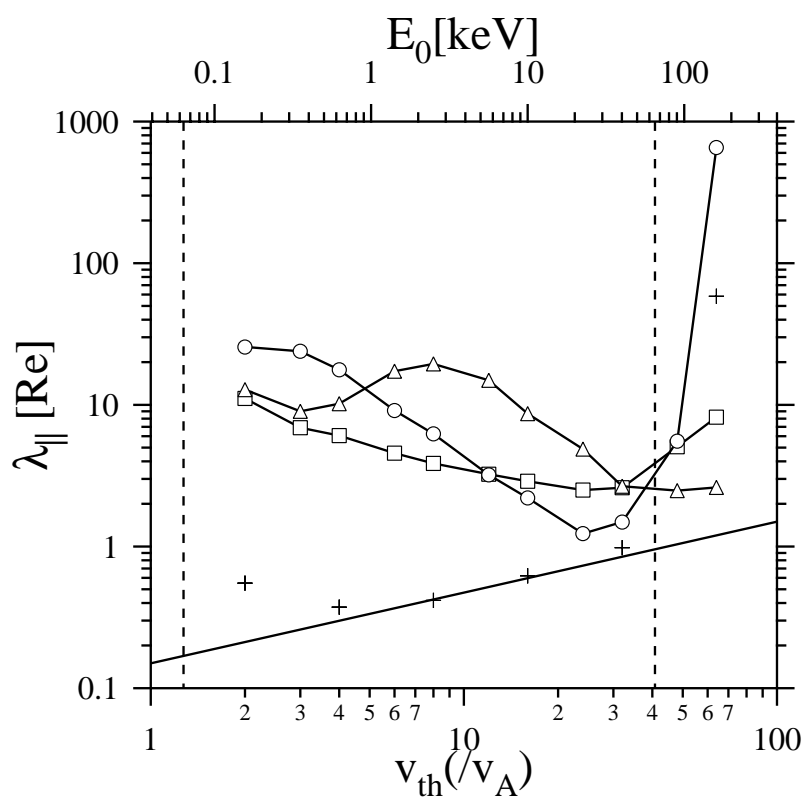

(b)

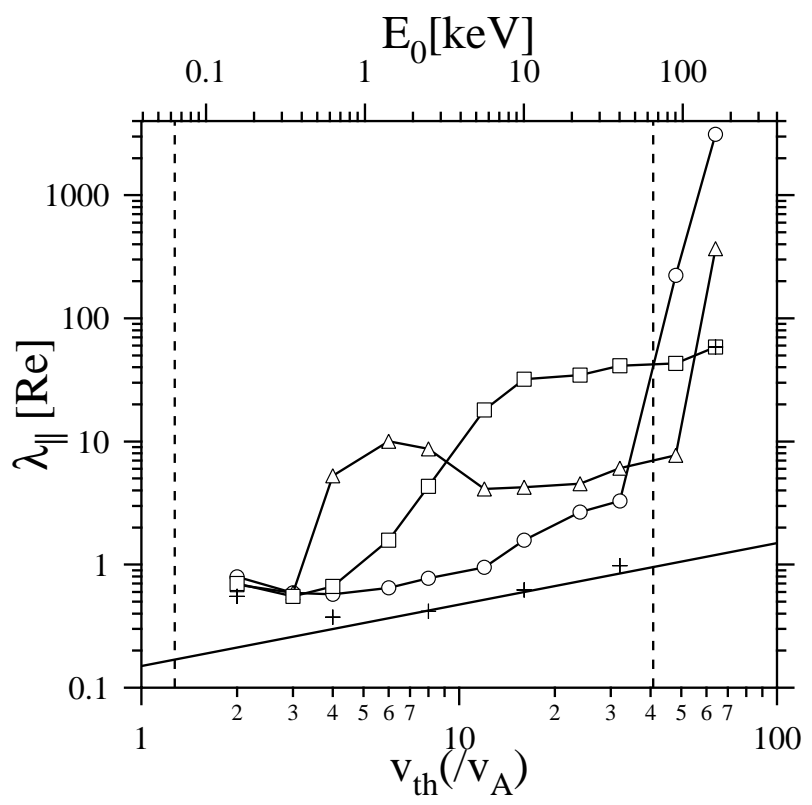

Fig. 7. Mean free path evaluated near the shock front for (a) regular cascade and (b) inverse cascade models. Symbols indicate the mean free paths obtained numerically with different plasma flow velocity, i.e., $u=2(\circ), u=8(\triangle)$, and $u=32(\square)$. In each panel, the symbols $(+)$ and solid line represent the numerical and the QLT values for homogeneous turbulence model, respectively. The vertical dashed lines in each panel represent the maximum and minimum resonence velocities (see text in 3.3.1). for $v_{t h}=2$. The numerical simulations done by Hada et al. (2003b) have shown that, when turbulence level is larger than $\delta B / B_{0}=0.1$, particles traverse $90^{\circ}$ pitch angle for a short time scale, indicating a deviation from the QLT near $90^{\circ}$ pitch angle. However, our result shows that, for a long time scale, nearly-isotropic scattering occurs in the homogeneous turbulence model, and that the resultant parallel diffusion is well described by the QLT. Therefore, we conclude that the deviation from QLT for a short time scale is not sensitive to the isotropic parallel diffusion for a long time scale.

Also, we note the effect of waves with high wavenumbers in the turbulence. In our model, the power spectrum falls zero at mode number higher than $m=128$. Thus, within the QLT, the pitch angle scattering near $90^{\circ}$ are expected to be inefficient, since the wavenumber, at which the particle near $90^{\circ}$ resonate linearly, is very large. However, the simulation shows efficient scattering across $90^{\circ}$ even though the higher wave modes are absent, implying the actual motion of the particle near $90^{\circ}$ is affected by a higher order terms, such as mirroring and resonance broadening, which are ignored in the QLT. This result is consistent with the conclusions of Giacalone and Jokipii (1999).

3.3.3 Dependence on the thermal velocity in the two cascade models

On the other hand, the numerical $\lambda_{\|}$are found to be larger than the value of the QLT, reaching the maximum at $\sim 30$ $R_{E}$ and $\sim 40 R_{E}$ in the regular and the inverse cascade models, respectively. In the regular cascade model $\lambda_{\|}$decreases with increasing $v_{t h}$, whereas in the inverse cascade model $\lambda_{\|}$ increases with increasing $v_{t h}$. In the regular cascade model, there are no waves to be in resonance with the particles at low energies in the far upstream region, and these particles reach the shock without efficient scattering. As the thermal velocity increases, the particles can resonate with the waves in the far upstream region, and they are convected toward the shock with efficient scattering. Hence $\lambda_{\|}$at the shock approaches the QLT value for increased $v_{t h}$.

On the contrary, in the inverse cascade model, there are resonant waves for the low energy particle in the far upstream region, and thus $\lambda_{\|}$at the shock for small $v_{t h}$ is close to the QLT value. As the thermal velocity increases, some particles in the tail of the Maxwellian distribution travel with own initial velocity toward the shock without efficient scattering by the waves. Hence $\lambda_{\|}$at the shock is larger than the QLT value as $v_{t h}$ is larger.

\subsubsection{Dependence on the plasma flow velocity in the two cascade models}

Let us study dependence on the plasma flow velocity $u$. In the regular cascade model, there is no clear dependence of $\lambda_{\|}$on $u$. The numerical $\lambda_{\|}$approaches the QLT value, as $u$ increases. When $u$ is small, the $\lambda_{\|}$increases toward the 
shock as shown in Fig. $6 \mathrm{~b}$. Whereas, when $u$ is large, the $\lambda_{\|}$ decreases toward the shock, and an efficient scattering based on the QLT occurs, since the particles convected with the flow quickly reach the turbulent shock.

In the inverse cascade model, $\lambda_{\|}$is larger than the QLT value as $u$ increases. When $u$ is small $(u=2)$, some particles with low energies are scattered by the waves far upstream, and other particles with high energies escape further upstream, $x<0$. As $u$ increases, we observed that the probability for particles to escape decreases, since more particles quickly reach the turbulent shock before escaping. Hence, the particles even with high energies in the upstream region travel toward the shock without experiencing efficient scattering by the waves assumed in the QLT.

\subsection{Thermal energy at the shock}

Figure 8 shows the variance of velocity defined by Eq. (8) for the regular and inverse cascade models. The bottom and top axes are the same as in Fig. 7, and the variance $\sigma_{v v}$ is in keV. The solid line shows the initial thermal energy $\left(E_{0}\right)$ equal to the particle thermal energy at the shock. The symbols represent the numerical $\sigma_{v v}$ evaluated at the shock and correspond to different plasma flow velocities $u$. The symbol notations used are the same as in Fig. 7. We use the same evaluation times as were used for the results presented in Fig. 7.

For a lower thermal energy $\left(v_{t h} \leq 8\right)$ corresponding to $E_{0}<2.5 \mathrm{keV}$, we find finite particle acceleration in both cascade models. The thermal energy maximally increases up to $\sim 4.2$ times initial thermal energy for $u=32$ and $v_{t h}=2$ in both cascade models. The particle acceleration occurs due to the additional electric fields, which are caused by spatial amplifications of transverse magnetic fluctuations. The acceleration is larger for a higher flow velocity, since the additional electric field $\Delta \mathbf{E}$ increases with increasing $u$. The maximum amplitudes of $\Delta \mathbf{E}$ are $0.05,0.2$, and 0.8 for $u=2,8$, and 32, respectively.

On the other hand in the higher thermal energy $\left(v_{t h}>8\right)$, the decreases of $\sigma_{v v}$ from the initial values are found in both models. These decreases are apparent in the inverse cascade model than in the regular cascade model. In the inverse cascade model, the thermal energy decreases more for lower flow velocity. These decreases are due to escaping particles. In the inverse cascade model and low flow velocity, some particles which belong to the high energy tail of the Maxwellian distribution are escaping from the upstream region. The variance $\sigma_{v v}$ is evaluated for the particles convected by the flow except for escaping particles, and the remaining particles belong to the lower energy part in the Maxwellian distribution. Therefore, by taking an ensemble average of these remaining particles, the evaluated $\sigma_{v v}$ at the shock is reduced from the initial values.

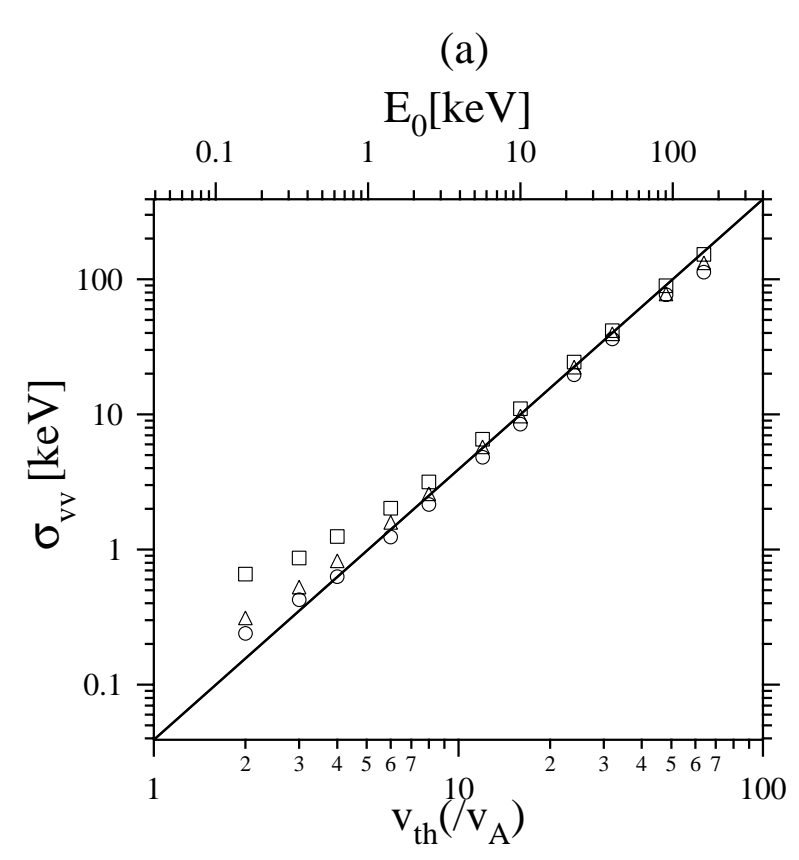

(b)

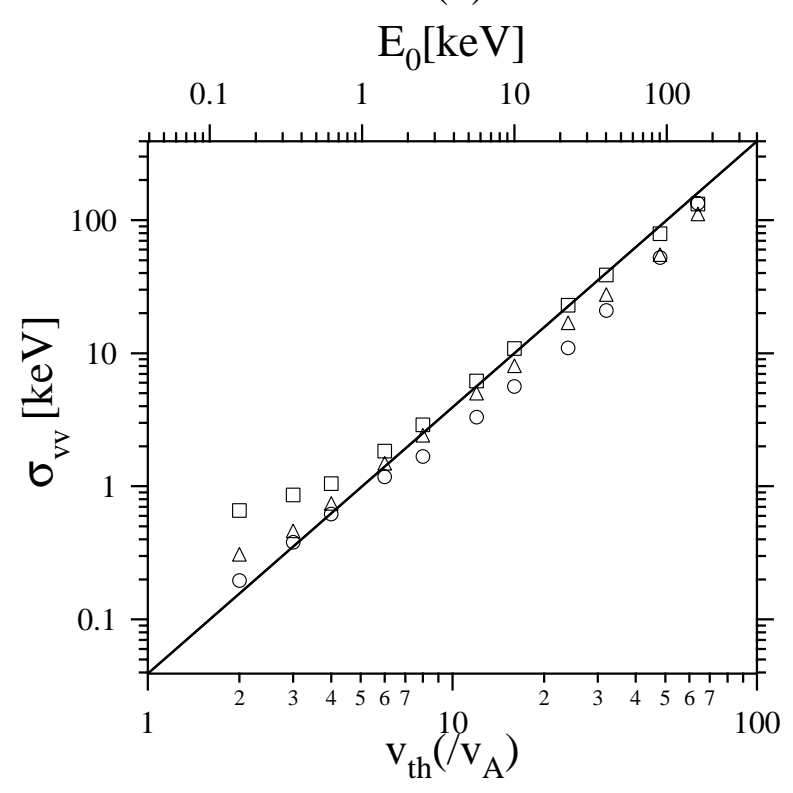

Fig. 8. Similar to Fig. 7 but the variance of velocity in Eq. (8) for (a) the regular cascade and (b) the inverse cascade models. Symbol notations are the same as in the Fig. 7.

\section{Summary and discussion}

\subsection{Summary}

We have studied the parallel (field-aligned) diffusion of energetic particles in the spatially evolving MHD turbulence with test particle simulations. We have considered the upstream 
region of the bow shock, up to 160 Earth radii from the shock, with the thermal energy of protons from $0.157 \mathrm{keV}$ to $160 \mathrm{keV}$. We have assumed that the bow shock has parallel shock geometry, and that MHD wave turbulence convected by the solar wind toward the shock is purely transverse in the one-dimensional system with a constant background magnetic field. We have employed two energy cascade models for the MHD turbulence: the regular cascade and the inverse cascade. In addition, the homogeneous turbulence model is used for comparison with the two cascade models. We have focused on the spatial dependence of the mean free path $\lambda_{\|}$of the particles due to the wave cascading. In these three models the particles are convected with the solar wind, and thus the spatially dependent $\lambda_{\|}$can be evaluated by a transformation of the time dependent $\lambda_{\|}$, where the spatial coordinate is assumed to be in the co-moving frame with the solar wind. We numerically computed particle orbits, and evaluated $\lambda_{\|}$as a function of time by taking an ensemble average of the orbits to obtain spatial dependence of $\lambda_{\|}$. We compare the numerical $\lambda_{\|}(x)$ with the theoretical $\lambda_{\|}(x)$ based on the QLT (quasi-linear theory). We have showed that spatial amplification of the magnetic field fluctuations creates transverse electric fields other than the motional electric field in the upstream region of the shock. We discuss acceleration of the particles due to these electric fields.

The principal results of this investigation are listed below:

1. Typical particle orbits are qualitatively different in the three turbulence models. In the homogeneous turbulence model, particle motion along the mean field is the Brownian motion due to random and isotropic scattering across the 90 degree pitch angle. For the regular cascade model, the motion is non-Brownian and is composed of "walk" (moving along the mean field with a constant velocity close to the resonance velocity) and "sticking" (stay a position for some time) segments. This motion is due to the trapping and de-trapping (release) by a wave with low wavenumber. In the inverse cascade model, the motion is combination of "walk" and the Brownian motion. This is due to anisotropic scattering caused by weak scattering around $0^{\circ}$ or $180^{\circ}$ pitch angles.

2. In the homogeneous turbulence model, the numerical $\lambda_{\|}$is independent of distance from the shock. On the other hand, in the two cascade models, the $\lambda_{\|}$depends on the distance. It also depends on the parameters such as the thermal velocity of the particles $v_{t h}$, solar wind flow velocity $u$, and wave cascade models.

3. We studied values of $\lambda_{\|}$at the shock for the different turbulence models when the thermal velocity satisfies the linear wave resonance condition. For the homogeneous turbulence model, the mean free path at the shock $\left(\lambda_{\|}<1 R_{E}\right)$ matches well the QLT result. On the other hand, for the two cascade models, $\lambda_{\|}$at the shock is found to be larger than the value of the QLT, reaching the maximum at $\sim 30 R_{E}$ and $\sim 40 R_{E}$ for the regular and inverse cascade models, respectively.

4. In the homogeneous turbulence model, $\lambda_{\|}$at the shock is $\lambda_{\|} \sim v_{t h}^{0.5}$ for the wave power spectrum index $\gamma=1.5$. This result is consistent with the QLT. On the other hand, $\lambda_{\|}$at the shock decreases or increases with the increasing $v_{t h}$ for the regular or the inverse cascade models, respectively. These results reflect evolution of the power spectrum in the two cascade models. Namely, waves with higher (lower) wavenumbers are absent far upstream in the regular (inverse) cascade model. That leads to inefficient scattering of the particles, depending on their thermal energies. In addition, in both cascade models, $\lambda_{\|}$at the shock depends on solar wind velocity.

5. In both cascade models, finite accelerations are found for particles at low thermal energies, typically, in the range less than $2.5 \mathrm{keV}$. The acceleration occurs due to the transverse electric field (with the motional electric field removed). The acceleration efficiency is estimated by the ratio of the thermal energy to the initial thermal energy $E_{0}$, which is about 4.2 for $E_{0}=0.157 \mathrm{keV}$ and $u=1600 \mathrm{~km} / \mathrm{s}$.

\subsection{Comparison with the theoretical $\lambda_{\|}^{Q L}(x)$ in Eq. (10)}

The observed spatial dependence of $\lambda_{\|}$is not consistent with the result of Eq. (10) based on the QLT. This discrepancy can be explained by the following. In the theory, a spatial evolution of the wave energy $\delta B^{2}$ is considered, but that of the wave power spectrum is not. In our simulation, the latter plays an aimportant role in the diffusion process. Even though the wave energy is the same, the pitch angle scattering of particles is qualitatively different, depending on which mode number the power spectrum has. When waves with low wavenumbers are only present, there is a coherent scattering due to trapping by the waves. Inversely, when waves with high wavenumbers are only present two scenarios are possible. There is an anisotropic scattering and no scattering due to lack of the waves to be in resonance with particles at high energy. These coherent and anisotropic scatterings produce the walk motion along the mean field for finite time. Hence, the spatial dependence of $\lambda_{\|}(x)$ cannot be described only by the spatial evolution of $\delta B^{2}$. Based on our results, one can estimate the parameter $\beta$, which models the spatial dependence of $\lambda_{\|}$in the empirical approach by Li et al. (2003).

\subsection{Effect of the solar wind velocity variations on the mean free path}

The observations show a large variation in the magnitudes of $\lambda_{\|}$from one event to another in a range of two orders of magnitude (Droge, 2000). In our study for the inverse cascade model, the $\lambda_{\|}$at the shock varies by more than an order 
of magnitude, if the solar wind velocity varies up to the factor of 16. It is generally observed that the solar wind velocity fluctuates from one event to another. Therefore, we conclude that the solar wind velocity is one of the important parameters to determine the value of $\lambda_{\|}$in presence of spatially evolving MHD waves.

For a homogeneous turbulence, the solar wind velocity does not affect $\lambda_{\|}$, since the $\lambda_{\|}$is not changed in the comoving solar wind frame. Namely, the turbulence statistics in the solar wind frame is uniquely determined as spatiallyindependent. Accordingly, the spatial and temporal scales characterizing the parallel diffusion, which are mean free path $\lambda_{\|}$and pitch angle relaxation time $\tau$, are also uniquely defined by the turbulence statistics. On the other hand, when MHD waves evolve spatially generating inhomogeneous turbulence, the foreshock size $L$ and the convection time T, at which the solar wind sweeps the foreshock region and which is written as $T=L / u$, become important as spatial and temporal scales. When the solar wind velocity is small, i.e., $\tau \ll T$, the particles will be isotropically scattered before reaching the shock if the waves to scatter them are present. Then, we predict that a slower solar wind leads to nearly isotropic scattering of the particles, and that it leads to a short mean free path described by the QLT. The prediction is consistent with our numerical result for the inverse cascade model (Fig. 7b). Namely, when $u$ decreases, the $\lambda_{\|}$ decreases and approaches to the QLT result. Another important aspect is that $\tau$ also has a spatial dependence. Since the turbulence is not evolved enough in the far upstream region, $\tau$ will be larger than that near the turbulent shock. The spatial dependence of $\tau$ should also be taken into account in understanding the parallel diffusion in the foreshock region. We suggest that a self-consistent model of wave-particle interaction beyond the QLT is needed for the case when two important time scales, i.e., the convection time and pitch angle relaxation time are of the same order.

\subsection{Comparison with numerical simulations of Giacalone} (2004)

The spatially evolving magnetic field fluctuations in our inverse cascade model are consistent with those in the hybrid simulations done by Giacalone (2004). We compared our numerical $\lambda_{\|}$with that in his study. In the paper by Giacalone (2004), the spatially dependent mean free path was obtained from fitting of a spatial profile of the energetic particle density and by assuming the exponential decay of a parallel diffusion coefficient. At the shock the mean free path, of the Table 2 in his paper, slightly increases from $\sim 6 R_{E}$ to $\sim 6.7$ $R_{E}$ in the energy range of $8.3 \mathrm{keV}<E<166 \mathrm{keV}$, where unit length $c / \omega_{i}$ and unit energy $E_{p}=m u^{2} / 2$ are assumed $100 \mathrm{~km}$ and $0.83 \mathrm{keV}$ for solar wind velocity $u=400 \mathrm{~km} / \mathrm{s}$, respectively. In our study (see Fig. 7b), we also observed a slight increase in $\lambda_{\|}$up to $\sim 5 R_{E}$ with $v_{t h}$ increase in the energy range of $5.6 \mathrm{keV}<E_{0}<40 \mathrm{keV}$ for $u=400 \mathrm{~km} / \mathrm{s}$. Therefore, the dependence of $\lambda_{\|}$at the shock on the thermal energy is consistent with that in Giacalone (2004).

In the result of Giacalone (2004), however, the simulated $\lambda_{\|}$increases with distance from the shock, and the $\lambda_{\|}$far upstream ( $\sim 320 R_{E}$ from the shock) increases to more than $230 R_{E}$. On the contrary in our inverse cascade model (see Fig. 6c), the numerical $\lambda_{\|}$slightly decreases with distance from the shock, and the $\lambda_{\|}$far upstream $\left(\sim 130 R_{E}\right.$ from the shock) is less than $\sim 10 R_{E}$. Hence, the spatial dependence of $\lambda_{\|}$in our result is inconsistent with that in the paper by Giacalone (2004). This inconsistency is due to the different methods of estimating $\lambda_{\|}$. In our study we took an ensemble average only for particles convected with the solar wind, whereas in Giacalone's study the escaping particles far upstream are also included in the fitting of the spatial density profile of the energetic particles.

The important feature of our method is that the $\lambda_{\|}(x)$ can be determined without any assumptions of the shape of $\lambda_{\|}(x)$ as a function of space. In the method employed by Giacalone (2004), the functional form of $\lambda_{\|}(x)$ should be needed to find the solution of the particle density profile and to fit the numerical profile with the obtained solution. Therefore, our method may contribute to the modification of the DSA (diffusive shock acceleration) process discussed by Giacalone (2004), by estimating a $\lambda_{\|}$with an arbitrary dependence on the spatial coordinate. Applying our method to the DSA process, we should vary the initial parameters for the particles such as injection position, which affects the probability of particles to escape far upstream, and we should also employ the initial velocity distribution like power-law type for shock accelerated particles.

\subsection{Pre-acceleration for the particles at low energies}

In addition, we suggest that particle acceleration considered in this paper might act as a pre-acceleration mechanism for the DSA process at a shock. It is essential for the DSA process that the particles can be scattered efficiently by the MHD waves upstream and downstream of the shock. The so-called injection problem addresses how particles might be accelerated from the thermal pool up to an energy where they can be diffused back and forth between the upstream and downstream regions. The present study reveals that solar wind particles at low energies can be accelerated before reaching the shock. The pre-acceleration is due to the transverse electric field with motional field extracted, which is caused by amplification of the MHD waves. For comparison with the observations, we should apply the real parameters to describe MHD fluctuations at the bow shock. In the cascade models, the growth rates of the magnetic fluctuations are arbitrary, that is, the growth length $\sim 2 R_{E}$ is comparable to the maximum wave length in the system. The growth length affects magnitude of the transverse electric field, which plays an important role in the acceleration process we observed. 


\section{Conclusions}

We investigated spatially dependent diffusion of the solar wind protons due to the wave cascading in the foreshock region, and calculated the mean free path $\lambda_{\|}$along background magnetic field as a function of space and thermal velocity. We found that results of the homogeneous turbulence model match predictions of the QLT (Quasi-Linear Theory). On the other hand, our results for the two cascading wave turbulence models are inconsistent with the QLT. Spatial evolution of wave power turbulent spectrum induces a non-Brownian particle motion due to trapping by a wave with low wavenumber and the anisotropic pitch angle scattering for a finite time scale. The numerical $\lambda_{\|}$are much larger than the values predicted by the QLT near the bow shock. We showed generation of transverse electric fields (with motional electric field removed) due to the wave-wave interactions. These electric fields can result in pre-acceleration of protons upstream of the bow shock. Further studies such as a self-consistent modeling and statistical analysis of a non-Brownian motion are needed for understanding of the observed $\lambda_{\|}$and modifications to the DSA (Diffusive Shock Acceleration) in the presence of evolving MHD turbulence in the foreshock region.

Acknowledgements. This work was partially supported by Grantin-Aid 17340146 and 17GS0208 for Creative Scientific Research "The Basic Study of Space Weather Prediction" of the Ministry of Education, Science, Sports and Culture of Japan. We would like to thank T. Hada, S. Matsukiyo, Y. Kuramitsu, and D. Koga for useful comments.

Edited by: B. Tsurutani

Reviewed by: two anonymous referees

\section{References}

Beeck, J., Mason, G. M., Hamilton, D. C., Wibberenz, G., Kunow, H., Hovestadt, D., and Klecker, B.: A multispacecraft study of the injection and transport of solar energetic particles, Astrophys. J., 322, 1052-1072, 1987.

Bieber, J. W., Matthaeus, W. H., and Smith, C. W.: Proton and electron mean free paths: The palmer consensus revisited, Astrophys. J., 420, 294-306, 1994.

Dröge, W.: The Rigidity Dependence of Solar Particle Scattering Mean Free Paths, Astrophys. J., 537, 1073-1079, 2000.

Eastwood, J. P., Lucek, E. A., Mazelle, C., Meziane, K., Narita, Y., Pickett, J., and Treumann, R. A.: The Foreshock, Space Sci. Rev. 118, 41-94, 2005.

Giacalone, J. and Jokipii, J. R.: The transport of cosmic rays across a turbulent magnetic field, Astrophys. J., 520, 204-214, 1999.

Giacalone, J.: Large-Scale Hybrid Simulations of Particle Acceleration at a Parallel Shock, Astrophys. J., 609, 452-458, 2004.

Gordon, B. E., Lee, A. M., Möbius, E., and Trattner, K. J.: Coupled hydromagnetic wave excitation and ion acceleration at interplanetary traveling shocks and Earth's bow shock revisited, J. Geophys. Res., 104, 28 263-28 278, 1999.
Hada, T, Koga, D. and Yamamoto, E.: Phase coherence of MHD waves in the solar wind, Space Sci. Rev. 107, 463-466, $2003 \mathrm{a}$.

Hada, T., Otsuka, F., Kuramitsu Y., and Tsurutani B. T.: Pitch Angle Diffusion of Energetic Particles by Large Amplitude MHD Waves, Proceedings of the 28th International Cosmic Ray Conference, 3709, 2003b.

Hoppe, M. M., Russell, C. T., Frank, L. A., Eastman, T. E., and Greenstadt, E. W.: Upstream hydromagnetic waves and their association with backstreaming ion populations - ISEE 1 and 2 observations, J. Geophys. Res., 86, 4471-4492, 1981.

Jokipii, J. R.: Cosmic-Ray Propagation. I. Charged Particles in a Random Magnetic Field, Astrophys. J., 146, 480, 1966.

Kennel, C. F. and Petschek, H. E.: Limit on stably trapped particle fluxes, J. Geophys. Res., 71, 1-28, 1966.

Kennel, C. F., Scarf, F. L., Coroniti, F. V., Russell, C. T., Wenzel, K.-P., Sanderson, T. R., van Nes, P., Smith, E. J., Tsurutani, B. T., and Scudder, J. D.: Plasma and energetic particle structure upstream of a quasi-parallel interplanetary shock, J. Geophys Res., 89, 5419-5435, 1984a.

Kennel, C. F., Edmiston, J. P., Russell, C. T., Scarf, F. L., Coroniti, F. V., Smith, E. J., Tsurutani, B. T., Scudder, J. D., Feldman, W. C., and Anderson, R. R.: Structure of the November 12, 1978, quasi-parallel interplanetary shock, J. Geophys. Res., 89, 54365452, 1984 b.

Koga, D. and Hada, T.: Phase coherence of foreshock MHD waves: wavelet analysis, Space Sci. Rev. 107, 495-498, 2003.

Koga, D., Chian, A. C.-L., Miranda, R. A., and Rempel, E. L.: Intermittent nature of solar wind turbulence near the Earth's bow shock: Phase coherence and non-Gaussianity, Phys. Rev. E, 75, 46 401-46 404, 2007.

Kuramitsu, Y., Krasnoselskikh, V.: Acceleration of charged particles by gyroresonant surfing at quasi-parallel shocks, A\&A, 438, 391-402, 2005.

Lee, M. A.: Coupled hydromagnetic wave excitation and ion acceleration upstream of the earth's bow shock, J. Geophys. Res., 87, 5063-5080, 1982.

Li, G.,Zank, G. P. and Rice, W. K. M.: Energetic particle acceleration and transport at coronal mass ejection-driven shocks, J. Geophys. Res., 108(A2), 1082, doi:10.1029/2002JA009666, 2003.

Li, Gang, Zank, G. P. and Rice, W. K. M.: Acceleration and transport of heavy ions at coronal mass ejection-driven shocks, J. Geophys. Res., 110, A06104, doi:10.1029/2004JA010600, 2005.

Meziane, K., Mazelle, C., D’Uston, C., Rème, H., Lin, R. P., Carlson, C. W., Larson, D., McFadden, J. P., Ergun, R. E., Anderson, K. A., Parks, G. K., Berdichevsky, D., and Lepping, R. P.: Wind observation of gyrating-like ion distributions and low frequency waves upstream from the earth's bow shock, Adv. Space. Res., 20, 703-706, 1997

Meziane, K., Mazelle, C., Lin, R. P., LeQuéau, D., Larson, D. E., Parks, G. K., and Lepping, R. P.: Three-dimensional observations of gyrating ion distributions far upstream from the Earth's bow shock and their association with low-frequency waves, J. Geophys. Res., 106(A4), 5731, doi:10.1029/2000JA900079, 2001

Nariyuki, Y. and Hada, T.: Remarks on nonlinear relation among phases and frequencies in modulational instabilities of parallel propagating Alfvén waves, Nonlin. Processes Geophys., 13, 425-441, 2006

Sanderson, T. R., Reinhard, R., van Nes, P., Wenzel, K.-P., Smith, 
E. J., and Tsurutani, B. T.: Observations of 35- 10 1600-keV protons and low-frequency waves upstream of interplanetary shocks, J. Geophys. Res., 90, 3973-3980, 1985

Schlickeiser, R.: Cosmic Rays Astrophysics, Springer-Verlag Berlin Heidelberg New York, 2002

Tsurutani, B. T. and Rodriguez, P: Upstream waves and particles: An overview of ISEE results, J. Geophys. Res., 86, 4319-4324, 1981

Tsurutani, B. T., Smith, E. J., Matsumoto, H., Brinca, A. L., and Omidi, N.: Highly nonlinear magnetic pulses at comet Giacobini-Zinner, Geophys. Res. Lett., 17, 757-760, 1990

Tsurutani, B. T. and Lakhina, G. S.: Some basic concepts of waveparticle interactions in collisionless plasmas Rev. Geophys., 35 , 491-501, 1997

Tsurutani, B. T., Zhang, L. D., Mason, G. L., Lakhina, G. S., Hada, T., Arballo, J. K., and Zwickl, R. D.: Particle transport in $3 \mathrm{He}-$ rich events: wave-particle interactions and particle anisotropy measurements, Ann. Geophys., 20, 427-444, 2002
Tsurutani, B. T., Lakhina, G. S., Pickett, J. S., Guarnieri, F. L., Lin, N., and Goldstein, B. E.: Nonlinear Alfvén waves, discontinuities, proton perpendicular acceleration, and magnetic holes/decreases in interplanetary space and the magnetosphere: intermediate shocks?, Nonlin. Processes Geophys., 12, 321-336, $2005 \mathrm{a}$

Tsurutani, B. T., Guarnieri, F. L., Lakhina, G. S., and Hada, T.: Rapid evolution of magnetic decreases (MDs) and discontinuities in the solar wind: ACE and Cluster, Geophys. Res. Lett., 32, $10103-10106,2005 b$

Wilkinson W. P.: The Earth's quasi-parallel bow shock: Review of observations and perspectives for Cluster, Planetary and Space Sci., 51, 629-647, 2003

Zank, G. P., Rice, W. K. M., and Wu, C. C.: Particle acceleration and coronal mass ejection driven shocks: A theoretical model, J. Geophys. Res., 105, 25 079-25 096, 2000 